\title{
Event-Triggered $H_{\infty}$ Control for Networked Control Systems with Time-Varying Delay
}

\author{
Huaicheng Yan, ${ }^{1}$ Sheng Yan, ${ }^{1}$ Hao Zhang, ${ }^{2}$ and Hongbo Shi ${ }^{1,2}$ \\ ${ }^{1}$ Key Laboratory of Advanced Control and Optimization for Chemical Processes of Ministry of Education and \\ the School of Information Science and Engineering, East China University of Science and Technology, Shanghai 200237, China \\ ${ }^{2}$ Department of Control Science and Engineering, Tongji University, Shanghai 200092, China
}

Correspondence should be addressed to Sheng Yan; yanyouth@126.com

Received 6 January 2014; Accepted 6 February 2014; Published 11 March 2014

Academic Editor: Xudong Zhao

Copyright (C) 2014 Huaicheng Yan et al. This is an open access article distributed under the Creative Commons Attribution License, which permits unrestricted use, distribution, and reproduction in any medium, provided the original work is properly cited.

\begin{abstract}
This paper deals with $H_{\infty}$ controller design problem for event-triggered networked control systems (NCSs), where the next task release time and finishing time are predicted based on the sampled states. A new model of NCSs that involves the network conditions, state, and event-triggered communication strategy is proposed. Based on this model, some novel criteria for the asymptotic stability analysis and $H_{\infty}$ state feedback controller design of the event-triggered NCSs with timevarying delay are established to guarantee a prescribed $H_{\infty}$ disturbance rejection attenuation level. Finally, a numerical example is provided to illustrate the effectiveness of the proposed method.
\end{abstract}

\section{Introduction}

NCSs are spatially distributed systems for which communication between sensors, actuators, and controllers is connected by a shared communication network. In recent years, NCSs have brought many innovative impacts on control systems. They are becoming increasingly important in industrial processes for many advantages, such as low installation and maintenance costs, high reliability, increased system flexibility, and decreased wiring and [1]. As such, network-based analysis and design have many industrial applications in, for example, aircrafts, manufacturing plants, robots, automobiles, and remote surgery [2-7]. However, great challenges are also met due to the network induced imperfection, namely, time delays, packet losses, disorder, time-varying transmission intervals, and competition of multiple nodes accessing networks as well as data quantization, which can deteriorate the performance of the NCSs and even destabilize the systems [3]. So far, much effort has been devoted to modeling, analysis, and design of NCSs in the presence of network-induced delays, packet dropouts, and disorder; see, for example, [8-14] and the references therein.
Notice that the network in NCSs is the shared bandlimited digital communication network [3]. One common problem to be addressed when considering NCSs is whether there is sufficient communication bandwidth to feedback information to the controller and then send the control commands to the actuators and the plant. Traditionally, the control task is executed periodically; this allows the closed-loop system to be analyzed and the controller to be designed using the well-developed theory on sampled-data systems [15]. However, the control strategy obtained based on this approach is conservative in the sense that resource usage is more frequent than necessary to ensure a specified performance level, since stability is guaranteed in the worst case scenarios under sufficiently fast periodic execution of the control action. To overcome this drawback, several researchers suggested the idea of event-triggered control. Event-triggered communication scheme has been proved to be an efficient way to reduce the transmitted data in the networks, which can relieve the burden of network bandwidth occupation in comparison with a traditional periodic sampling method. In [16], started from the paradigm that a realtime scheduler could be regarded as a feedback controller that decides which task is executed at any given instant, a simple 
event-triggered scheduler based on this feedback paradigm was investigated to guarantee performance thus relaxing the more traditional periodic execution requirements. In [17], a decentralized event-triggered implementation, over sensor/actuator networks, of centralized nonlinear controllers was presented. In [18], a new self-triggering scheme that ensures finite-gain $L_{2}$ stability of the resulting self-triggered feedback systems was proposed. This scheme relaxes the assumptions that the magnitude of the process noise is bounded by a linear function of the norm of the system state. In [19], a novel event-triggering scheme was presented to ensure exponential stability of the resulting sampleddata system. The scheme postpones the triggering of events over previously proposed methods and therefore enlarges the intersampling period. The control design problem of eventtriggered networked systems with both state and control input quantizations was addressed in [20]. An innovative delay system model was proposed, and the criteria for the asymptotical stability analysis and control synthesis of eventtriggered NCSs were established. Unfortunately, to the best of the authors' knowledge, up to now, the stabilization and $H_{\infty}$ control problems for general NCSs with simultaneous consideration of the network-induced time-varying delays and event-triggered communication scheme have not been adequately addressed yet, which still remains an interesting research topic. This motivates the current research.

In this paper, the $H_{\infty}$ state feedback controller design method for the event-triggered NCSs with time-varying delays is presented. Different from some existing ones, the feedback NCSs in this paper is modeled as a delay system considering the network-induced delays and event-triggering scheme. By using Lyapunov-Krasovskii function approach, some new sufficient conditions that guarantee the asymptotic stability of the closed-loop NCSs are established in terms of linear matrix inequities (LMIs). Moreover, the explicit expression of feedback gain is also derived with eventtriggering and network-induced delays. Finally, a simulation example is given to illustrate the effectiveness of the proposed method.

Notation. $\mathbb{R}^{n}$ and $Z^{+}$denote the $n$-dimensional Euclidean space and positive integer set, respectively. $\mathbb{R}^{m \times n}$ is the set of $m \times n$ real matrices. $\operatorname{Sym}\{X\}$ denotes the expression $X+X^{\top} . I_{n}$ denotes the $n \times n$ identity matrix. The notation $X>0$ (resp., $X \geq 0$ ) denotes a real symmetric positive definite (positive semidefinite). In symmetric block matrices, “*” is used as ellipsis for terms induced by symmetry; $\operatorname{diag}\{\cdots\}$ denotes the block-diagonal matrix. Matrixes, if not explicitly stated, are assumed to have appropriate dimensions.

\section{Problem Formulation}

Consider the NCSs with event-triggering shown in Figure 1. The physical plant is given by

$$
\begin{gathered}
\dot{x}(t)=A x(t)+B u(t)+B_{\omega} \omega(t), \\
z(t)=C x(t)+D u(t),
\end{gathered}
$$

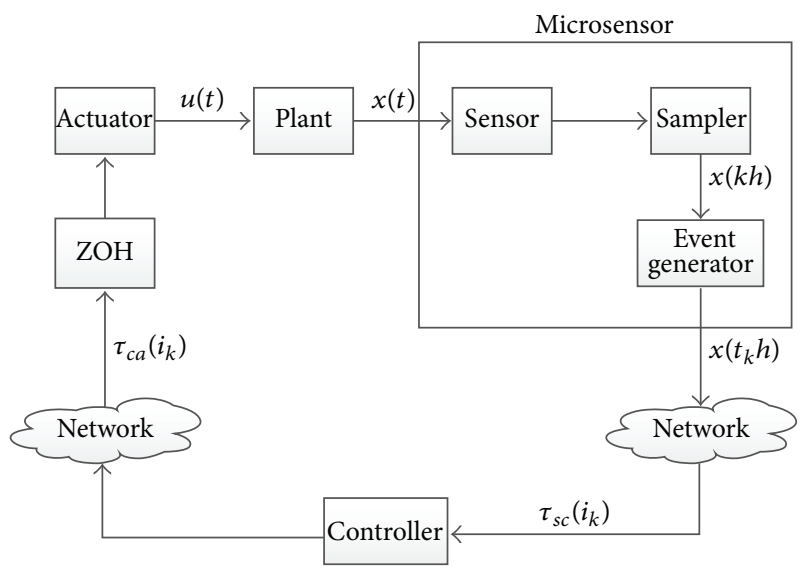

FIGURE 1: The structure of event-triggered NCSs with time-varying delay.

where $x(t) \in \mathbb{R}^{n}$ is the state vector, $u(t) \in \mathbb{R}^{m}$ is the control input vector, $\omega(t) \in L_{2}[0, \infty)$ is the disturbance input, and $z(t) \in \mathbb{R}^{p}$ is the control output vector, respectively. $A, B$, $B_{\omega}, C$, and $D$ are the parameter matrices with appropriate dimensions. The initial condition of the system (1) is given by $x\left(t_{0}\right)=x_{0}$. Throughout this paper, we assume that system (1) is controlled throughout a network with a networked state feedback controller, which is directly connected to the actuator through a zero-order holder $(\mathrm{ZOH})$ [21].

The purpose of this paper is to design a linear controller $u(t)=K x(t)$, where $K$ is a matrix to be determined later, such that the resulting closed-loop system satisfies the required $H_{\infty}$ performance.

To facilitate theoretical development, the following assumptions, which are common in NCSs research in open literature, are made in this paper.

Assumption 1. The sensors in the communication network are time-triggered with a constant sampling period $h$, while the controllers and actuators are event-triggered.

Assumption 2. The signal in a network is transmitted with a single packet, and the computational delay of the controller is negligible [22-25], and the data packet losses do not occur in transmission.

Assumption 3. The total network-induced delay $\tau_{k}\left(k \in Z^{+}\right)$ is bounded; that is, $0<\tau_{m} \leq \tau_{k} \leq \tau_{M}$, where $\tau_{m}$ and $\tau_{M}$ denote the lower and upper delay bounds, respectively [15, 21].

As depicted in (1), considering the limited capacity of the communication channels and also for reducing the data transmission rate in the network, we show a framework of the proposed event-triggered communication scheme for the NCSs. The event-triggered communication scheme can be expressed as [20]

$$
\varrho_{\gamma_{k+j} h}^{\top} V \varrho_{\gamma_{k+j} h} \geq \sigma x^{\top}\left(i_{k+j} h\right) V x\left(i_{k+j} h\right)
$$


where $\varrho_{\gamma_{k+j} h}=x\left(i_{k+j} h\right)-x\left(i_{k} h\right)$ is the error between the current sampling data $x\left(i_{k+j} h\right)$ and the latest transmitted sampling data $x\left(i_{k} h\right), V$ is a positive matrix, $j \in Z^{+}$, and $\sigma \in[0,1]$

Remark 4. The communication scheme (2) is characterized by the parameters $\sigma, V$, and $h$. Only the sampled state data $x\left(i_{k+j} h\right)$ that satisfy the quadratic condition will be transmitted to the controller. Obviously, this scheme will determine the load of the communication in the network. As a special case, if $\sigma=0$ in (2), inequality (2) holds for all the sampled state data $x\left(i_{k+j} h\right)$; this scheme will reduce to a timetriggered communication scheme.

Under communication scheme (2), we assume that the release times are $t_{0} h, t_{1} h, t_{2} h, \ldots$, where $t_{0} h$ is the initial time; $\gamma_{k} h=t_{k+1} h-t_{k} h$ denotes the transmission period of the event generator. Take the network-induced time-varying delay $\tau_{i_{k}}$ into consideration; these release signals will arrive at the controller side at the instants $t_{0} h+\tau_{0}, t_{1} h+\tau_{1}, t_{2} h+\tau_{2}, \ldots$, respectively.

Based on the above analysis, considering the effect of the time-varying delay in the communication network and the event-triggered communication scheme (2), for $t \in\left[t_{k} h+\right.$ $\left.\tau_{i_{k}}, t_{k+1} h+\tau_{i_{k+1}}\right)$, the system (1) under the control $u(t)=K x(t)$ can be described as

$$
\begin{gathered}
\dot{x}(t)=A x(t)+B u\left(t_{k} h\right)+B_{\omega} \omega(t), \\
z(t)=C x(t)+D u\left(t_{k} h\right), \\
u\left(t_{k} h\right)=K x\left(t_{k} h\right), \quad t \in\left[t_{k} h+\tau_{i_{k}}, t_{k+1} h+\tau_{i_{k+1}}\right) .
\end{gathered}
$$

Based on the above analysis, we consider the following intervals:

$$
\left[t_{k} h+\tau_{i_{k}}, t_{k+1} h+\tau_{i_{k+1}}\right) .
$$

It is easy to make the conclusion that $\gamma_{k} h \geq h$.

(1) If $\gamma_{k} h \leq h+\tau_{M}-\tau_{i_{k+1}}$, we define

$$
\tau(t)=t-t_{k} h, \quad t \in\left[t_{k} h+\tau_{i_{k}}, t_{k+1} h+\tau_{i_{k+1}}\right) .
$$

Furthermore, we define an error vector as

$$
\varrho_{k}(t)=0
$$

(2) If $\gamma_{k} h>h+\tau_{M}-\tau_{i_{k+1}}$, it can be easily shown that $l \geq 1$ exists such that

$$
l h+\tau_{M}-\tau_{i_{k+1}}<\gamma_{k} h \leq(l+1) h+\tau_{M}-\tau_{i_{k+1}} .
$$

Then the range $\left[t_{k} h+\tau_{i_{k}}, t_{k+1} h+\tau_{i_{k+1}}\right)$ can be divided into the following $l+1$ subranges:

$$
\begin{aligned}
& {\left[t_{k} h+\tau_{i_{k}}, t_{k+1} h+\tau_{i_{k+1}}\right)} \\
& =\left[t_{k} h+\tau_{i_{k}}, t_{k} h+h+\tau_{M}\right) \\
& \quad \cup\left\{\bigcup_{n=1}^{l-1}\left[t_{k} h+n h+\tau_{M}, t_{k} h+(n+1) h+\tau_{M}\right)\right\} \\
& \quad \cup\left[t_{k} h+l h+\tau_{M}, t_{k+1} h+\tau_{i_{k+1}}\right) .
\end{aligned}
$$

Define a function $\tau(t)$ as

$$
\tau(t)=\left\{\begin{array}{l}
t-t_{k} h \quad t \in\left[t_{k} h+\tau_{i_{k}}, t_{k} h+h+\tau_{M}\right), \\
t-t_{k} h-n h \\
\quad t \in \bigcup_{n=1}^{l-1}\left[t_{k} h+n h+\tau_{M}, t_{k} h+(n+1) h+\tau_{M}\right), \\
t-t_{k} h-l h \\
\quad t \in\left[t_{k} h+l h+\tau_{M}, t_{k+1} h+\tau_{i_{k+1}}\right) .
\end{array}\right.
$$

It follows from (9) that

$$
\tau_{m} \leq \tau(t) \leq h+\tau_{M} .
$$

At this time, we define the error vector as

$$
\varrho_{k}(t)=\left\{\begin{array}{l}
0 \quad t \in\left[t_{k} h+\tau_{i_{k}}, t_{k} h+h+\tau_{M}\right), \\
x\left(t_{k} h+n h\right)-x\left(t_{k} h\right) \\
\quad t \in \bigcup_{n=1}^{l-1}\left[t_{k} h+n h+\tau_{M}, t_{k} h+(n+1) h+\tau_{M}\right), \\
x\left(t_{k} h+l h\right)-x\left(t_{k} h\right) \\
\quad t \in\left[t_{k} h+l h+\tau_{M}, t_{k+1} h+\tau_{i_{k+1}}\right) .
\end{array}\right.
$$

Combining (6) and (11), it can be seen that

$$
\begin{array}{r}
\varrho_{k}^{\top}(t) V \varrho_{k}(t)<\sigma x^{\top}(t-\tau(t)) V x(t-\tau(t)), \\
t \in\left[t_{k} h+\tau_{i_{k}}, t_{k+1} h+\tau_{i_{k+1}}\right) .
\end{array}
$$

Combining (5), (6), (9), and (11) together with (3), define $h_{1}=\tau_{m}, h_{2}=\tau_{M}+h$; we can obtain the following closed-loop system as follows:

$$
\begin{gathered}
\dot{x}(t)=A x(t)+B K x(t-\tau(t))-B K \varrho_{k}(t)+B \omega(t), \\
t \in\left[t_{k} h+\tau_{i_{k}}, t_{k+1} h+\tau_{i_{k+1}}\right), \\
z(t)=C x(t)+D K x(t-\tau(t))-D K \varrho_{k}(t), \\
x(t)=\phi(t), \quad t \in\left[t_{0}-h_{2}, t_{0}-h_{1}\right),
\end{gathered}
$$

where we define $\phi(t)$ as the initial function of $x(t)$.

To make the theoretical development easier, the following definition will be used. 
Definition 5. The closed-loop system (13) is said to be asymptotically stable with an $H_{\infty}$ disturbance attenuation level $\gamma$; that is, (1) system (13) is asymptotically stable with $\omega(t) \equiv 0$. (2) Under zero initial condition, $\|z(t)\|_{2}<\gamma\|\omega(t)\|_{2}$, for any nonzero $\omega(t) \in L_{2}[0, \infty)$ and a prescribed $\gamma>0$.

\section{Main Results}

Firstly, we develop a stability criterion for the system (13) with time-varying communication delay. Then, Theorem 7 is presented which lays the foundation for the $H_{\infty}$ controller design.

Theorem 6. For some given parameters $h_{1}, h_{2}, \gamma, \sigma$, and feedback gain $K$, under the event-triggered communication scheme (2), the system (13) is asymptotically stable with an $\mathrm{H}_{\infty}$ performance index $\gamma$ for the disturbance attention, if there exist real matrixes $P>0, Q_{i}>0, L_{i}>0(i=1,2), V>0$, and $G$ with appropriate dimensions such that the following LMIs hold:

$$
\begin{gathered}
{\left[\begin{array}{cc}
\Sigma_{11} & * \\
\Sigma_{21} & \Sigma_{22}
\end{array}\right]<0,} \\
{\left[\begin{array}{cc}
L_{2} & * \\
G & L_{2}
\end{array}\right]>0,}
\end{gathered}
$$

where

$$
\begin{aligned}
& \Sigma_{11}=\left[\begin{array}{cccccc}
\Phi_{11} & * & * & * & * & * \\
\Phi_{21} & \Phi_{22} & * & * & * & * \\
\Phi_{31} & \Phi_{32} & \Phi_{33} & * & * & * \\
0 & \Phi_{42} & \Phi_{43} & \Phi_{44} & * & * \\
\Phi_{51} & 0 & 0 & 0 & \Phi_{55} & * \\
\Phi_{61} & 0 & 0 & 0 & 0 & \Phi_{66}
\end{array}\right] \\
& \Sigma_{21}=\left[\begin{array}{lll}
h_{1} \xi_{1}^{\top} L_{1}^{\top} & h \xi_{1}^{\top} L_{2}^{\top} & \xi_{2}^{\top}
\end{array}\right]^{\top}, \\
& \Sigma_{22}=\operatorname{diag}\left\{\begin{array}{lll}
-L_{1} & -L_{2} & -I
\end{array}\right\}, \\
& \Phi_{11}=P A+A^{\top} P+Q_{1}+Q_{2}-L_{1}, \quad \Phi_{21}=L_{1}, \\
& \Phi_{22}=-Q_{1}-L_{2}-L_{1}, \quad \Phi_{31}=K^{\top} B^{\top} P, \\
& \Phi_{32}=-G+L_{2}, \quad \Phi_{33}=\sigma V-2 L_{2}+G+G^{\top}, \\
& \Phi_{42}=G, \quad \Phi_{43}=-G+L_{2}, \quad \Phi_{44}=-Q_{2}-L_{2}, \\
& \Phi_{51}=-K^{\top} B^{\top} P, \quad \Phi_{55}=-V, \quad \Phi_{61}=B_{\omega}^{\top} P, \quad \Phi_{66}=-\gamma^{2} I, \\
& \xi_{1}=\left[\begin{array}{llllll}
A & 0 & B K & 0 & -B K & B_{\omega}
\end{array}\right] \text {, } \\
& \xi_{2}=\left[\begin{array}{llllll}
C & 0 & D K & 0 & -D K & 0
\end{array}\right] \text {, } \\
& h=h_{2}-h_{1}
\end{aligned}
$$

Proof. Construct a Lyapunov-Krasovskii functional candidate as

$$
\begin{aligned}
V(t)= & x^{\top}(t) P x(t)+\int_{t-h_{1}}^{t} x^{\top}(s) Q_{1} x(s) d s \\
& +\int_{t-h_{2}}^{t} x^{\top}(s) Q_{2} x(s) d s
\end{aligned}
$$

$$
\begin{aligned}
& +h_{1} \int_{-h_{1}}^{0} \int_{t+s}^{t} \dot{x}^{\top}(v) L_{1} \dot{x}(v) d v d s \\
& +h \int_{-h_{2}}^{-h_{1}} \int_{t+s}^{t} \dot{x}^{\top}(v) L_{2} \dot{x}(v) d v d s,
\end{aligned}
$$

where $P>0, V>0, Q_{j}>0$, and $L_{j}>0(j=1,2)$. Taking the derivation of $V(t)$ for $t \in\left[t_{k} h+\tau_{k}, t_{k+1} h+\tau_{k+1}\right)$ and by adding and subtracting the term $\varrho_{k}^{\top}(t) V \varrho_{k}(t)$, we have

$$
\begin{aligned}
\dot{V}(t)= & 2 x^{\top}(t) P \dot{x}(t)+x^{\top}(t) Q_{1} x(t) \\
& -x^{\top}\left(t-h_{1}\right) Q_{1} x\left(t-h_{1}\right)+x^{\top}(t) Q_{2} x(t) \\
& -x^{\top}\left(t-h_{2}\right) Q_{2} x\left(t-h_{2}\right)+h_{1}^{2} \dot{x}^{\top}(t) L_{1} \dot{x}(t) \\
& -h_{1} \int_{t-h_{1}}^{t} \dot{x}^{\top}(v) L_{1} \dot{x}(v) d v+h^{2} \dot{x}^{\top}(t) L_{2} \dot{x}(t) \\
& -h \int_{t-h_{2}}^{t-h_{1}} \dot{x}^{\top}(v) L_{2} \dot{x}(v) d v+\varrho_{k}^{\top}(t) V \varrho_{k}(t) \\
& -\varrho_{k}^{\top}(t) V \varrho_{k}(t)+z^{\top}(t) z(t)-z^{\top}(t) z(t) .
\end{aligned}
$$

Applying Jensen's inequality [26] and convex reciprocally approach [27] to deal with the integral items in (18), noticing (15), we obtain

$$
\begin{gathered}
-h_{1} \int_{t-h_{1}}^{t} \dot{x}^{\top}(v) L_{1} \dot{x}(v) d v \leq-\eta^{\top}(t) \Pi_{1} \eta(t), \\
-h \int_{t-h_{2}}^{t-h_{1}} \dot{x}^{\top}(v) L_{2} \dot{x}(v) d v \\
=-h\left[\int_{t-\tau(t)}^{t-h_{1}} \dot{x}^{\top}(v) L_{2} \dot{x}(v) d v\right. \\
\left.\quad+\int_{t-h_{2}}^{t-\tau(t)} \dot{x}^{\top}(v) L_{2} \dot{x}(v) d v\right] \\
\leq-\frac{h}{\tau(t)-h_{1}}\left[x^{\top}\left(t-h_{1}\right) L_{2} x\left(t-h_{1}\right)\right. \\
\leq-\eta^{\top}(t) \prod_{2} \eta(t), \\
-\frac{h}{h_{2}-\tau(t)}\left[x^{\top}(t-\tau(t)) L_{2} x(t-\tau(t))\right. \\
\left.-x^{\top}\left(t-h_{2}\right) L_{2} x\left(t-h_{2}\right)\right]
\end{gathered}
$$


with $\underline{\eta}^{\top}(t)=\left[x^{\top}(t) x^{\top}\left(t-h_{1}\right) x^{\top}(t-\tau(t)) x^{\top}(t-\right.$ $\left.\left.h_{2}\right) \varrho_{k}^{\top}(t) \omega^{\top}(t)\right]$,

$$
\begin{aligned}
\Pi_{1}= & {\left[\begin{array}{cccccc}
L_{1} & * & 0 & 0 & 0 & 0 \\
-L_{1} & L_{1} & 0 & 0 & 0 & 0 \\
0 & 0 & 0 & 0 & 0 & 0 \\
0 & 0 & 0 & 0 & 0 & 0 \\
0 & 0 & 0 & 0 & 0 & 0 \\
0 & 0 & 0 & 0 & 0 & 0
\end{array}\right], } \\
\Pi_{2}= & {\left[\begin{array}{cccccc}
0 & 0 & 0 & 0 & 0 & 0 \\
0 & L_{2} & * & * & 0 & 0 \\
0 & G-L_{2} & 2 L_{2}-G-G^{\top} & * & 0 & 0 \\
0 & -G & G-L_{2} & L_{2} & 0 & 0 \\
0 & 0 & 0 & 0 & 0 & 0 \\
0 & 0 & 0 & 0 & 0 & 0
\end{array}\right] . }
\end{aligned}
$$

Notice that $\Sigma_{21}^{\top} \Sigma_{22}^{-1} \Sigma_{21}=-\left[h_{1}^{2} \xi_{1}^{\top} L_{1} \xi_{1}+h^{2} \xi_{1}^{\top} L_{2} \xi_{1}+\xi_{2}^{\top} \xi_{2}\right]$, $\dot{x}(t)=\xi_{1} \eta(t)$, and $z(t)=\xi_{2} \eta(t)$; we have

$$
\begin{aligned}
\eta^{\top} & (t)\left[\Sigma_{21}^{\top} \Sigma_{22}^{-1} \Sigma_{21}\right] \eta(t) \\
= & -\eta^{\top}(t)\left[h_{1}^{2} \xi_{1}^{\top} L_{1} \xi_{1}+h^{2} \xi_{1}^{\top} L_{2} \xi_{1}+\xi_{2}^{\top} \xi_{2}\right] \eta^{\top}(t) \\
& =-h_{1}^{2} \dot{x}^{\top}(t) L_{1} \dot{x}(t)-h^{2} \dot{x}^{\top}(t) L_{2} \dot{x}(t)-z^{\top}(t) z(t) .
\end{aligned}
$$

Combining (12), (17), (19), and (21), we obtain

$$
\begin{aligned}
\dot{V}(t) \leq & \eta^{\top}(t)\left(\Sigma_{11}-\Sigma_{21}^{\top} \Sigma_{22}^{-1} \Sigma_{21}\right) \eta(t) \\
& -z^{\top}(t) z(t)+\gamma^{2} \omega^{\top}(t) \omega(t),
\end{aligned}
$$

where $\Sigma_{11}, \Sigma_{21}$, and $\Sigma_{22}$ are defined in (14).

By the Schur complements, the Lyapunov-Krasovskii function (14) guarantees that $\dot{V}(t)<0$ in (17); we can derive that the system $(13)$ with $\omega(t) \equiv 0$ is asymptotically stable and $\|z(t)\|_{2}<\gamma\|\omega(t)\|_{2}$ under zero initial condition. This completes the proof.

Now we are in a position to design the state feedback $H_{\infty}$ controller for the closed-loop system (13).

Theorem 7. For given parameters $h_{1}, h_{2}, \gamma$, and $\sigma$, under the event-triggered communication scheme (2), the system (13) is asymptotically stable with an $\mathrm{H}_{\infty}$ performance index $\gamma$ for the disturbance attention, and the feedback gain $K=Y X^{-1}$, if there exist real matrixes $X>0, \widetilde{Q}_{i}>0, \widetilde{L}_{i}>0(i=1,2), \widetilde{V}>0$, and $\widetilde{G}$ with appropriate dimensions such that the following matrix inequities hold:

$$
\begin{aligned}
& {\left[\begin{array}{cc}
\Sigma_{11}^{\prime} & * \\
\Sigma_{21}^{\prime} & \Sigma_{22}^{\prime}
\end{array}\right]<0,} \\
& {\left[\begin{array}{cc}
\widetilde{L}_{2} & * \\
\widetilde{G} & \widetilde{L}_{2}
\end{array}\right]>0,}
\end{aligned}
$$

where

$$
\begin{aligned}
& \Sigma_{11}^{\prime}=\left[\begin{array}{cccccc}
\widetilde{\Phi}_{11} & * & * & * & * & * \\
\widetilde{\Phi}_{21} & \widetilde{\Phi}_{22} & * & * & * & * \\
\widetilde{\Phi}_{31} & \widetilde{\Phi}_{32} & \widetilde{\Phi}_{33} & * & * & * \\
0 & \widetilde{\Phi}_{42} & \widetilde{\Phi}_{43} & \widetilde{\Phi}_{44} & * & * \\
\widetilde{\Phi}_{51} & 0 & 0 & 0 & \widetilde{\Phi}_{55} & * \\
\widetilde{\Phi}_{61} & 0 & 0 & 0 & 0 & \widetilde{\Phi}_{66}
\end{array}\right] \\
& \Sigma_{21}^{\prime}=\left[\begin{array}{llll}
h_{1} \tilde{\xi}_{1}^{\top} & h \tilde{\xi}_{1}^{\top} & \tilde{\xi}_{2}^{\top}
\end{array}\right]^{\top} \\
& \Sigma_{22}^{\prime}=\operatorname{diag}\left\{-X \widetilde{L}_{1}^{-1} X,-X \widetilde{L}_{2}^{-1} X,-I\right\} \text {, } \\
& \widetilde{\Phi}_{11}=X A+A^{\top} X+\widetilde{Q}_{1}+\widetilde{Q}_{2}-\widetilde{L}_{1}, \quad \widetilde{\Phi}_{21}=\widetilde{L}_{1} \text {, } \\
& \widetilde{\Phi}_{22}=-\widetilde{Q}_{1}-\widetilde{L}_{2}-\widetilde{L}_{1}, \quad \widetilde{\Phi}_{31}=Y^{\top} B^{\top}, \quad \widetilde{\Phi}_{32}=-\widetilde{G}+\widetilde{L}_{2}, \\
& \widetilde{\Phi}_{33}=\sigma \widetilde{V}-2 \widetilde{L}_{2}+\widetilde{G}+\widetilde{G}^{\top}, \quad \widetilde{\Phi}_{42}=\widetilde{G}, \\
& \widetilde{\Phi}_{43}=-\widetilde{G}+\widetilde{L}_{2}, \quad \widetilde{\Phi}_{44}=-\widetilde{Q}_{2}-\widetilde{L}_{2}, \quad \widetilde{\Phi}_{51}=-Y^{\top} B^{\top}, \\
& \widetilde{\Phi}_{55}=-\widetilde{V}, \quad \Phi_{61}=B_{\omega}^{\top}, \quad \widetilde{\Phi}_{66}=-\gamma^{2} I, \\
& \widetilde{\xi}_{1}=\left[\begin{array}{llllll}
A X & 0 & B Y & 0 & -B Y & B_{\omega}
\end{array}\right], \\
& \tilde{\xi}_{2}=\left[\begin{array}{llllll}
C X & 0 & D Y & 0 & -D Y & 0
\end{array}\right], \\
& h=h_{2}-h_{1} \text {. }
\end{aligned}
$$

Proof. Define $X=P^{-1}, X Q_{i} X=\widetilde{Q}_{i}, X L_{i} X=\widetilde{L}_{i}(i=$ $1,2), X V X=\widetilde{V}, X G X=\widetilde{G}$, and $Y=K X$, and pre- and postmultiply (14), (15) with $\operatorname{diag}\left\{X, X, X, X, X, I, L_{1}^{-1}, L_{2}^{-1}, I\right\}$, $\operatorname{diag}\{X, X\}$, and their transposes, respectively. By Schur complement, we can obtain (23) and (24) from (14) and (15). Therefore, we can know from Theorem 6, (23), and (24) that the system (13) is asymptotically stable with an $H_{\infty}$ performance index $\gamma$ for the disturbance attention.

Remark 8. Theorem 7 provides a useful way of codesign for both the state feedback controller gain and the eventtriggered parameter by solving a set of LMIs in (23). However, the derived matrix inequalities cannot be solved directly by making use of the MATLAB LMI control toolbox due to the nonlinear terms such as $X V X$ in (23). To reduce the conservatism that may result from the driving LMIs based on (23), one can apply the cone complementarity linearization (CCL) algorithm [28]. The information of the transmission delay is also involved in (23). So our design method can be used to deal with the case with network transmission delay. For given condition on the time-varying delay, by solving (23), the state feedback gain matrix $K$ and triggered constant $V$ can be obtained, which can be used to guarantee the required $H_{\infty}$ performance.

\section{Illustrative Example}

In this section, a numerical example is provided to validate the effectiveness of the theoretical results. The inverted 
pendulum introduced by [18] is considered. The plant's statespace representation is given by

$$
\dot{x}(t)=\left[\begin{array}{cccc}
0 & 1 & 0 & 0 \\
0 & 0 & -\frac{m g}{M} & 0 \\
0 & 0 & 0 & 1 \\
0 & 0 & \frac{g}{l} & 0
\end{array}\right] x(t)+\left[\begin{array}{c}
0 \\
\frac{1}{M} \\
0 \\
-\frac{1}{M l}
\end{array}\right] u(t) .
$$

We choose other parameter matrices as

$$
\begin{gathered}
C=\left[\begin{array}{llll}
1 & 1 & 1 & 1
\end{array}\right]^{\top}, \quad D=0.1, \quad B_{\omega}=C^{\top}, \\
\omega(t)= \begin{cases}\operatorname{sgn}(\sin t) & \text { if } t \in[0,10] \\
0 & \text { otherwise, }\end{cases}
\end{gathered}
$$

where $M=10$ is the cart mass and $m=1$ is the mass of the pendulum bob, $l=3$ is the length of the pendulum arm, and $g=10$ is the gravitational acceleration. The initial state is chosen as $x_{0}=[0.98,0,0.2,0]^{\top}$. As we can see, the eigenvalues of the system matrices are $0,0,1.8257$, and -1.8257 ; thus the system is unstable without a controller. Applying Theorem 7 with $\sigma=0.1, \gamma=200, h_{1}=0.01$, and $h_{2}=0.11$, the corresponding feedback gain and the triggered matrix are obtained as

$$
\begin{aligned}
K & =\left[\begin{array}{llll}
-1.9739 & -9.7238 & 53.0167 & 34.9453
\end{array}\right], \\
V & =\left[\begin{array}{cccc}
70.8218 & 58.5581 & 23.0154 & 32.7182 \\
58.5581 & 88.5920 & 8.3766 & 28.5856 \\
23.0154 & 8.3766 & 171.6515 & 103.9430 \\
32.7182 & 28.5856 & 103.9430 & 91.7903
\end{array}\right] .
\end{aligned}
$$

Taking the sampling period $h=0.1 \mathrm{~s}$, the release instants and release intervals of system (26) are shown in Figure 2. The state response of system (26) with feedback controller gain (28) and event-triggered communication scheme (29) are depicted in Figure 3, respectively. Figure 3 shows that the system state converges to zero.

\section{Conclusion}

To reduce the communication load in the network, a novel event-triggered scheme has been proposed to determine when the sampling signal data will be transmitted. An eventtriggered $H_{\infty}$ control design method has been proposed for NCSs with time-varying delay. A delay system model has been used to describe the prosperities of the event trigger and effects of the transmission delay on the system. Based on this model, new criteria for stability with an $H_{\infty}$ norm bound and $H_{\infty}$ control design are developed. Since the relationship between the network-induced delay, the state feedback controller gain, and the trigger parameters are established, it can be used to schedule NCSs resources through adjusting one or more parameters for a better tradeoff between the control performance and the network conditions. The numerical example is given to demonstrate the effectiveness of the proposed algorithm.

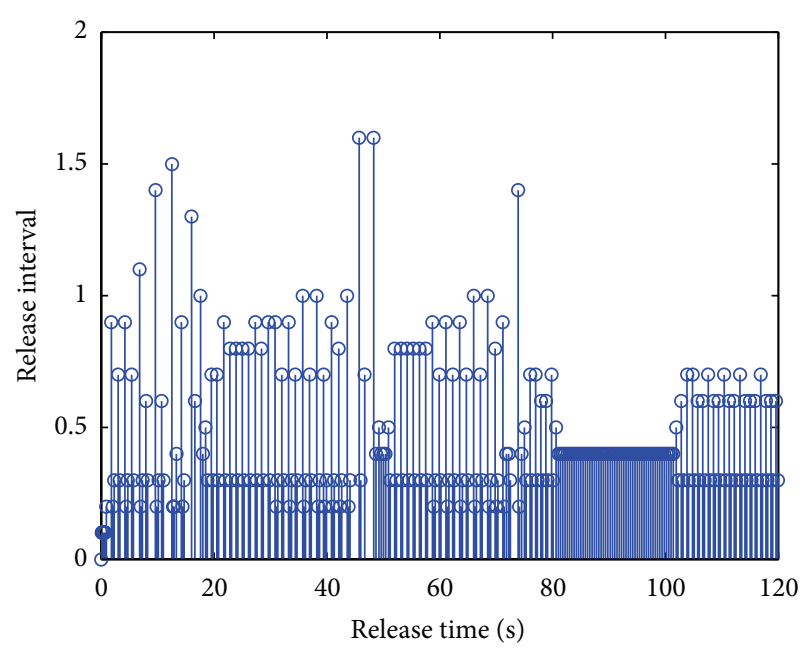

FIgURE 2: The release instants and release interval.

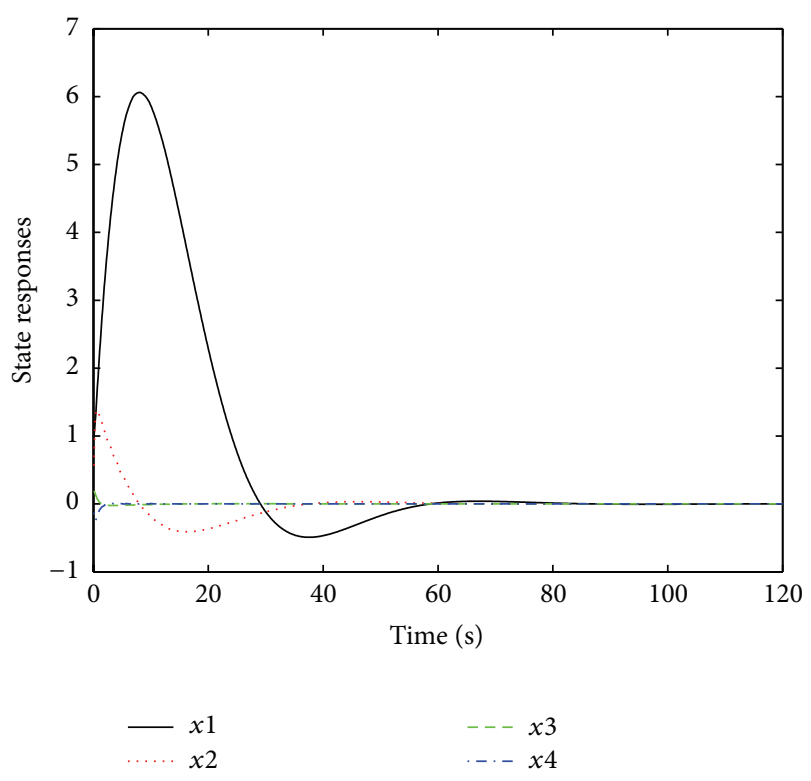

FIGURE 3: The state response of system.

\section{Conflict of Interests}

The authors declare that there is no conflict of interests regarding the publication of this paper.

\section{Acknowledgment}

This work is supported by the National Natural Science Foundation of China (61004028, 61272064, and 61273026), the Innovation Program of Shanghai Municipal Education Commission (12zz052), and the Fundamental Research Funds for the Central Universities. 


\section{References}

[1] L. G. Bushnell, "Networks and control," IEEE Control Systems Magazine, vol. 21, no. 1, pp. 22-23, 2001.

[2] R. Gupta and M. Chow, "Networked control system: overview and research trends," IEEE Transactions on Industrial Electronics, vol. 57, no. 7, pp. 2527-2535, 2010.

[3] J. P. Hespanha, P. Naghshtabrizi, and Y. Xu, "A survey of recent results in networked control systems," in Proceedings of the 45th IEEE International Conference on Decision and Control, pp. 138$162,2007$.

[4] Y. Tipsuwan and M. Chow, "Control methodologies in networked control systems," Control Engineering Practice, vol. 11, no. 10, pp. 1099-1111, 2003.

[5] C. Meng, T. Wang, W. Chou, S. Luan, Y. Zhang, and Z. Tian, "Remote surgery case: robot-assisted teleneurosurgery," IEEE International Conference on Robotics and Automation, vol. 1, pp. 819-823, 2004.

[6] S. Yin, S. Ding, and H. Luo, "Real-time implementation of fault tolerant control system with performance optimization," IEEE Transactions on Industrial Electronics, vol. 61, no. 5, pp. 24022411, 2014.

[7] H. Li, H. Gao, and P. Shi, "New passivity analysis for neural networks with discrete and distributed delays," IEEE Transactions on Neural Networks, vol. 21, no. 11, pp. 1842-1847, 2010.

[8] L. X. Zhang, H. J. Gao, and O. Kaynak, "Network-induced constraints in networked control systems-a survey," IEEE Transactions on Industrial Informatics, vol. 9, no. 1, pp. 403-416, 2013.

[9] H. J. Gao, T. W. Chen, and J. Lam, "A new delay system approach to network-based control," Automatica, vol. 44, no. 1, pp. 39-52, 2008.

[10] H. Zhang, H. C. Yan, F. W. Yang, and Q. J. Chen, "Quantized control design for impulsive fuzzy networked systems," IEEE Transactions on Fuzzy Systems, vol. 19, no. 6, pp. 1153-1162, 2011.

[11] H. C. Yan, Z. Z. Su, H. Zhang, and F. W. Yang, "Observerbased $H_{\infty}$ control for discrete-time stochastic systems with quantisation and random communication delays," IET Control Theory \& Applications, vol. 7, no. 3, pp. 372-379, 2013.

[12] H. Zhang, H. C. Yan, T. Liu, and Q. J. Chen, "Fuzzy controller design for nonlinear impulsive fuzzy systems with time-delay," IEEE Transactions on Fuzzy Systems, vol. 19, no. 5, pp. 844-856, 2011.

[13] K. Y. You and L. H. Xie, "Survey of recent progress in studying networked control systems," Acta Automatica Sinica, vol. 39, no. 2, pp. 101-117, 2013.

[14] T. C. Yang, "Networked control system: a brief survey," IET Control Theory and Applications, vol. 153, no. 4, pp. 403-412, 2006.

[15] D. Yue, Q.-L. Han, and J. Lam, "Network-based robust $H_{\infty}$ control of systems with uncertainty," Automatica, vol. 41, no. 6 , pp. 999-1007, 2005.

[16] P. Tabuada, "Event-triggered real-time scheduling of stabilizing control tasks," IEEE Transactions on Automatic Control, vol. 52, no. 9, pp. 1680-1685, 2007.

[17] M. Mazo, Jr. and P. Tabuada, "Decentralized event-triggered control over wireless sensor/actuator networks," IEEE Transactions on Automatic Control, vol. 56, no. 10, pp. 2456-2461, 2011.

[18] X. F. Wang and M. D. Lemmon, "Self-triggered feedback control systems with finite-gain $L_{2}$ stability," IEEE Transactions on Automatic Control, vol. 54, no. 3, pp. 452-467, 2009.
[19] X. F. Wang and M. D. Lemmon, "On event design in eventtriggered feedback systems," Automatica, vol. 47, no. 10, pp. 2319-2322, 2011.

[20] S. L. Hu and D. Yue, "Event-triggered control design of linear networked systems with quantizations," ISA Transactions, vol. 51, no. 1, pp. 153-162, 2012.

[21] Y. Xu and J. P. Hespanha, "Optimal communication logics in networked control systems," in Proceedings of the 43th IEEE Conference on Decision and Control, vol. 4, pp. 3527-3532, 2004.

[22] D. Yue, E. Tian, and Q.-L. Han, "A delay system method for designing event-triggered controllers of networked control systems," IEEE Transactions on Automatic Control, vol. 58, no. 2, pp. 475-481, 2013.

[23] X. Jia, D. Zhang, X. Hao, and N. Zheng, "Fuzzy $H_{\infty}$ tracking control for nonlinear networked control systems in TS fuzzy model," IEEE Transactions on Systems, Man, and Cybernetics B, vol. 39, no. 4, pp. 1073-1079, 2009.

[24] C. Peng and Y.-C. Tian, "Networked $H_{\infty}$ control of linear systems with state quantization," Information Sciences, vol. 177, no. 24, pp. 5763-5774, 2007.

[25] H. K. Lam and L. D. Seneviratne, "LMI-based stability design of fuzzy controller for nonlinear systems," IET Control Theory \& Applications, vol. 1, no. 1, pp. 393-401, 2007.

[26] K. Gu, J. Chen, and V. L. Kharitonov, Stability of Time Delay Systems, Springer, 2003.

[27] P. Park, J. W. Ko, and C. Jeong, "Reciprocally convex approach to stability of systems with time-varying delays," Automatica, vol. 47, no. 1, pp. 235-238, 2011.

[28] L. E. Ghaoui, F. Oustry, and M. AitRami, "A cone complementarity linearization algorithm for static output-feedback and related problems," IEEE Transactions on Automatic Control, vol. 42, no. 8, pp. 1171-1176, 1997. 


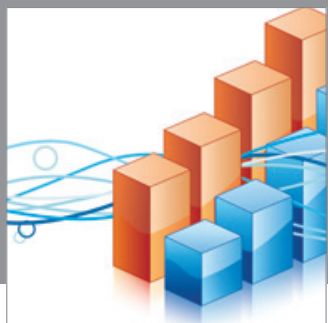

Advances in

Operations Research

mansans

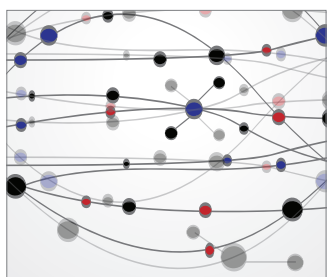

The Scientific World Journal
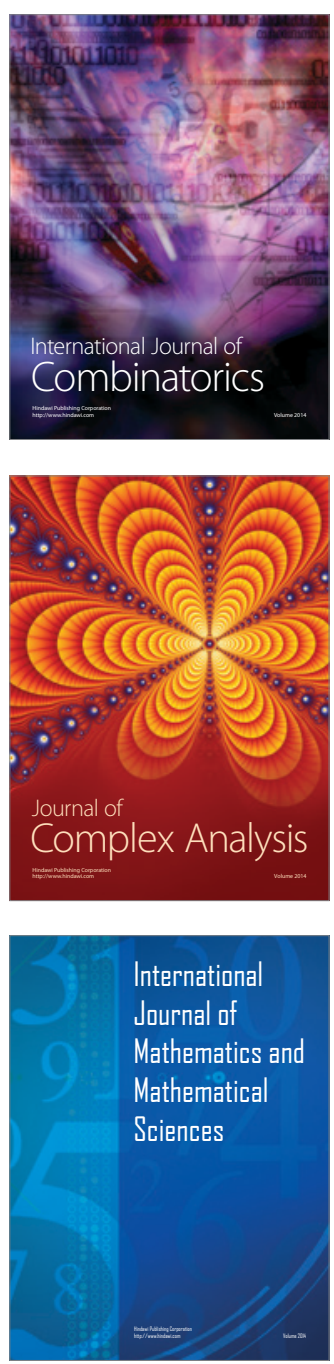
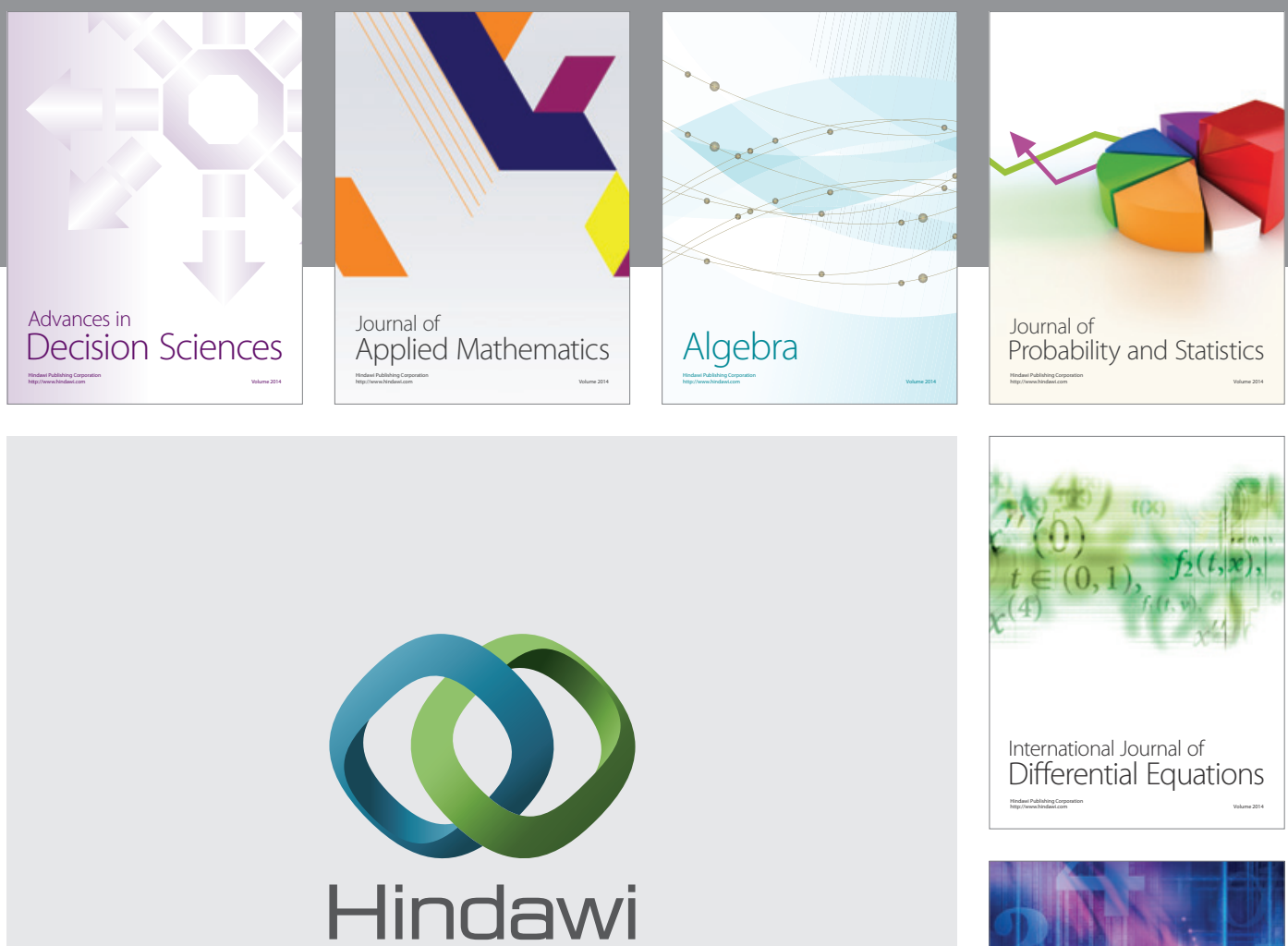

Submit your manuscripts at http://www.hindawi.com
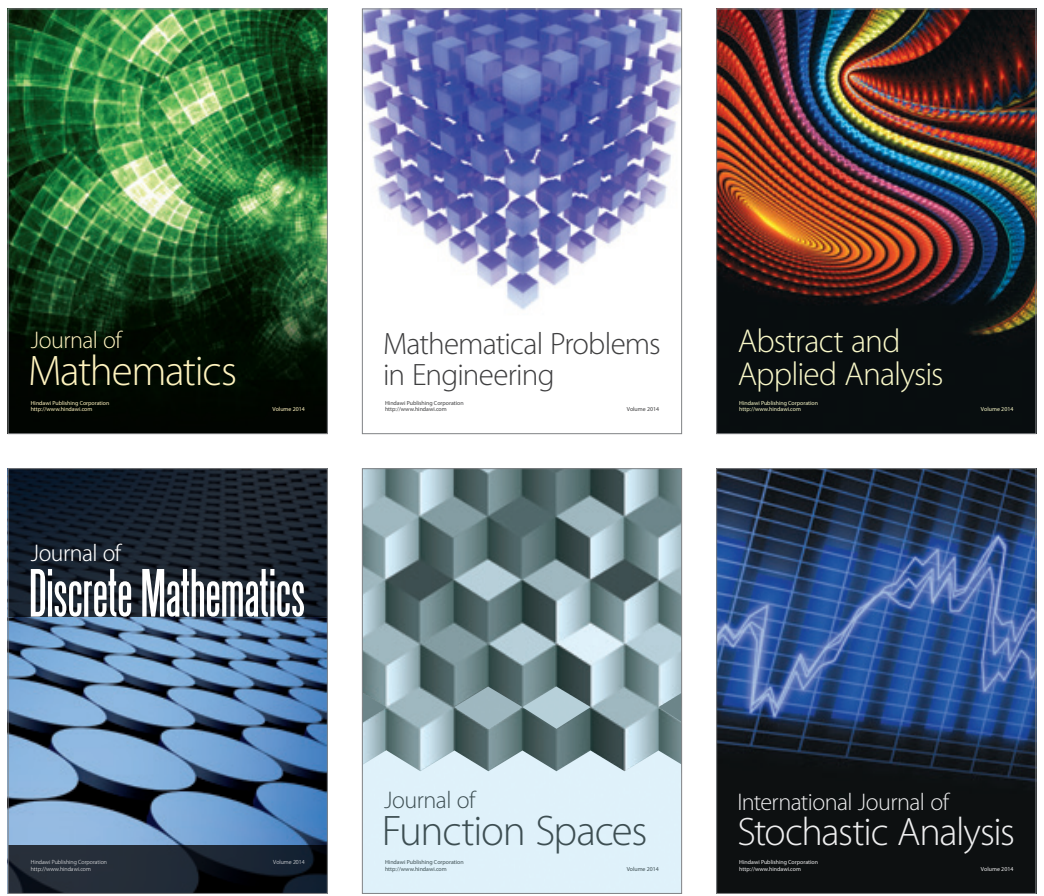

Journal of

Function Spaces

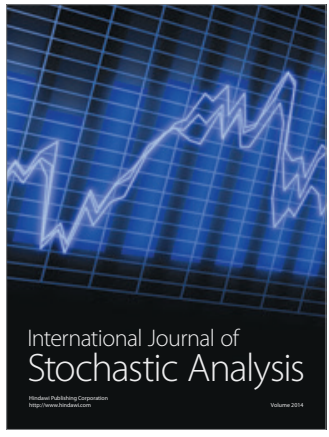

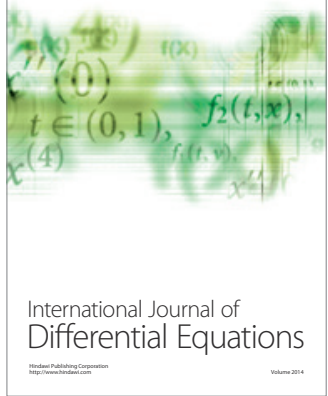
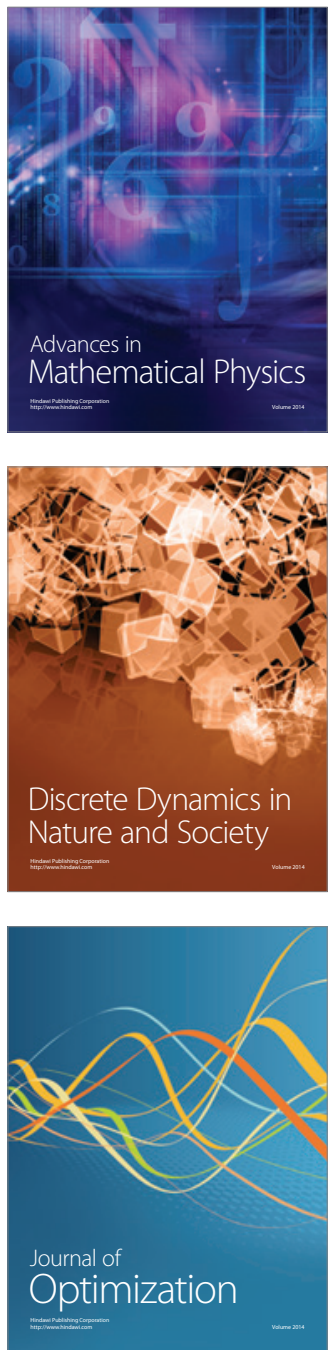